\title{
Homology modeling of apoprotein Opsin and covalent docking of 11-cis retinal and 11-cis 3, 4-didehyroretinal to obtain structures of Rhodopsin and Porphyropsin from Zebra danio, Danio rerio (Hamilton, 1822)
}

\author{
Susmita Dey ${ }^{1}$, Gitartha Kaushik ${ }^{2}$, Saurav Mahanta ${ }^{3}$ and Arijit Chakraborty ${ }^{4, *}$ \\ ${ }^{1}$ Dept. of Zoology, Royal Global University, Guwahati, Assam, India- 781035; ${ }^{2}$ Faulty of Science, Assam Downtown University, Assam, \\ India - 781026; ${ }^{3}$ Bioinformatics Center, National Institute of Electronics and Information Technology, Assam, India-781008; ${ }^{4}$ Department \\ of Sports \& Exercise Science, Somaiya Sports Academy, Somaiya Vidyavihar University, Vidyanagar, Mumbai, Maharashtra 400077, India
}

Received: April 6, 2020; Revised: Sept 20, 2020; Accepted: Nov 10, 2020

\begin{abstract}
Opsin proteins are classically seven transmembrane receptor proteins which detect light. The present investigation describes the stereoscopic structure of the apoprotein Opsin by online structure determining tools using the crystallographic structure of Rhodopsin from Bos taurus as the template. The modeled structure was validated and checked through validated data tools for stereochemical quality of a protein structure, ProSa and the square root of the mean square deviation between the templates and the predicted structure was calculated using PyMol. It was found that the chromophores, 11-cis retinal and 11cis 3, 4-didehydroretinal was covalently docked with apoprotein which gives the structures of Rhodopsin and Porphyropsin respectively. Due to its close homology to the human rhodopsin (accession P08100), zebra fish rhodopsin may serve as an excellent model for study of human diseases in future. This article represents the test of structure/function relationship of apoprotein opsin in this ornamental aquarium fish and provides a foundation for future work exploring cellular and molecular pathways of photoreception in retinal development and disease models.
\end{abstract}

Keywords: Zebra fish, Opsin, Rhodopsin, Porphyropsin, 11-cis retinal, 11-cis 3,4-didehyroretinal

\section{Introduction}

Studies have shown the use of visual pigments by representatives of almost all the vertebrates, where visual pigments are Rhodopsin and Porphyropsin. Both rod visual pigments contain the same apoprotein Opsin; however, they are covalently linked to different groups. Rhodopsin binds 11-cis-retinal, whereas Porphyropsin is linked to 11cis 3, 4-didehydroretinal (La Franco et al., 2018; Ganong 2005). These pigments possess various spectral characteristics like maximum absorbance and absorbency index (Marschall, et al., 2012). The absorption maxima of Rhodopsin are nearly $500 \mathrm{~nm}$, whereas Porphyropsin is in the range of $520-535 \mathrm{~nm}$. It has been found that the visual pigments may vary in response to light, temperature, and other environmental stimuli (Korenyak and Govardovskii, 2013).

Studies suggest that certain fish retinas comprise a visual purple pigment rather than the contemporary Rhodopsin of red - color which was confirmed spectrophotometrically (Enright et al., 2015). Similar studies suggested that Porphyropsin a purple rod-pigment, is a characteristic of freshwater fishes, whereas the red rod visual pigment, Rhodopsin is characteristic of terrestrial craniate and most of the Sea fishes. It has also been found that many freshwater fishes have Porphyropsin and/or admixtures of Rhodopsin and Porphyrins (Corush., 2019; Toyama et al., 2008, Ochuko et. al., 2014).

The organism Danio rerio was selected for the current investigation as its visual system shares high similarities with other vertebrates (Gestri et al., 2012; Golsmith and Harris 2003). Preliminary studies on $D$. rerio suggested that it possesses only Rhodopsin as visual pigment (Morrow and Chang 2015, Cameron 2002; Chinen et.al., 2003), whereas in a later study it has been confirmed that they have a paired visual system involving both Rhodopsin and Porphyropsin (Allison et.al., 2004).

In the present scenario, computational method such as homology modeling has been used to bridge the gap between sequence information and structures. The current investigation represents 3D models of Rhodopsin and Porphyropsin by homology modeling and docking methods for $D$. rerio which can be used to study the biochemical mechanism underlying the working of both the visual pigments.

\section{Materials and Methods}

\subsection{Protein sequence analysis}

Protein sequence analysis including, physicochemical parameters, molecular weight, sum of cationic and anionic residues, theoretical isoelectric point (pI), absorbency index (Porterfield and Zlotnick, 2010), aliphatic index

\footnotetext{
* Corresponding author e-mail: say.arijit22@gmail.com, arijit@somaiya.edu
} 
(Sahay and Shakya, 2010), Grand Average hydropathy (GRAVY) (Wanyonyi et.al., 2011), half-life (Sharma et.al., 2014) and instability index (Gamage et.al., 2019) was calculated using the Expasy's ProtParam prediction server (Gasteiger et al., 2003).

\subsection{Prediction of the three-dimensional structure}

The protein sequence of the apoprotein from the organism $D$. rerio was obtained from the UniProt database (accession number: P35359). The sequence was analyzed using the $G$ protein coupled receptor- Sequence-structurefeature-extractor (GPCR -SSFE) database (Worth et. al., 2011). Further, a protein BLAST was also carried out (Altschul et.al., 1990). After obtaining the template, homology modeling was carried out using MODELLER $9 \mathrm{v} 8$ software. It makes use of a command interpreter for designing stereochemical structures of proteins along with their confounders by consummation of spatial restraints (Sali and Blundell 1993). The reliability of the model was analyzed by three (3) ways: (i) PROCHECK (Laskowski et.al., 1993), (ii) by calculation of the RMSD (root mean square deviation) between the template and the predicted structure using PyMol (version 1.8, Schrödinger, Inc, USA), and (iii) using ProSa (Wiederstein and Sippl 2007).

\subsection{Covalent docking}

The receptor and the ligand structures were prepared with Glide and Prime. The Protein Preparation Wizard was used for preparing the receptor, and LigPrep was used for preparing the ligands (Reddy and Vanga 2017). A maximum of 10 poses per ligand are established and are subject to minimization of post-docking. The apoprotein Opsin is covalently bonded to 11-cis-retinal and 11-cis 3, 4-didehydroretinal to form structures of Rhodopsin and Porphyropsin respectively. Covalent docking to the nitrogen of Lysine 296 was performed using the Prime module of Schrodinger. The covalent docking facility allows the selection of the attachment point to the receptor and the possible attachment point on the ligand and then runs a prime loop prediction to find all feasible poses for the ligand.

\section{Results and Discussion}

In the current study, the sequence of Opsin from $D$. rerio has been repossessed from the UniProt database. The predicted physiochemical characteristic of Opsin showed that the protein has a molecular weight of $39.7064 \mathrm{kDa}$. Opsin has 20 (Arg + Lys) amino acid composition of positively charged and 22 (Asp + Glu) residues that are negatively charged. This also correlates with more number of negatively charged residues present in Opsin. The coefficient extinction of Opsin at $280 \mathrm{~nm}$ is $69175 \mathrm{M}^{-1} \mathrm{~cm}^{-}$

${ }^{1}$ in lieu of the concentration of amino acids Phenylalanine, Trptophan and Tyrosine. It has been well established that for a protein is stable if the measured instability index is smaller than 40 and corresponding value above 40 foresees that the protein may be virtually unstable (Sahay and Shakya, 2010). The instability index of Opsin was found to be 46.09 , which indicate that the protein is unstable in vitro. The aliphatic index for the protein sequence was found to be 85.90, which indicated thermo stability (Ashokan et. al., 2010). The GRAVY value computed for carotenoid binding protein was 0.457. Molecular orientation using Ramachandran plot showed the modelled structure ha most of the residues (88.6\%) in the fully allowed region, $11.1 \%$ in the additionally allowed region, $0.3 \%$ in the generously allowed region and nothing in the prohibited region.

\subsection{Assessment of the three-dimensional structure}

The sequence of the apoprotein from $D$. rerio was analyzed by the GPCR - SSFE database. Current result suggests the structure of Rhodopsin from organism Bos taurus (Protein data bank, PDB ID -1U19) as the template for the query sequence. The reasons for the selection of the template are presented in table 1.

Table 1. Reasons for the selection of the template

\begin{tabular}{|c|c|c|c|}
\hline Helix & Template & $\begin{array}{l}\text { Sequence } \\
\text { Similarity }\end{array}$ & Reason for template choice \\
\hline TMH1 & $1 \mathrm{U} 19$ & 89.7 & Has Pro in same position \\
\hline TMH2 & $1 \mathrm{U} 19$ & 100.0 & $\begin{array}{l}\text { Has Gly-Gly motif in same } \\
\text { position }\end{array}$ \\
\hline ТМHЗ & $1 \mathrm{U} 19$ & 91.4 & $\begin{array}{l}\text { No insertion or 2nd disulphide } \\
\text { bridge and the highest sequence } \\
\text { similarity to suggested template }\end{array}$ \\
\hline TMH4 & $1 \mathrm{U} 19$ & 92.0 & $\begin{array}{l}\text { No second disulphide bridge } \\
\text { between TMH3 and ECL2, no } \\
\text { insertion and highest sequence } \\
\text { similarity to suggested template }\end{array}$ \\
\hline TMH5 & $1 \mathrm{U} 19$ & 96.3 & $\begin{array}{l}\text { No intra-ECL2 disulphide bridge, } \\
\text { no disulphide bridge between } \\
\text { ECL1-ECL2, no sequence } \\
\text { similarity to the TMH5 extension } \\
\text { of sRHO }\end{array}$ \\
\hline TMH6 & $1 \mathrm{U} 19$ & 100.0 & $\begin{array}{l}\text { No disulphide bridge between } \\
\text { TMH6 and ECL3, no sequence } \\
\text { similarity to the TMH6 helix } \\
\text { extension of sRHO and highest } \\
\text { sequence similarity to suggested } \\
\text { template }\end{array}$ \\
\hline TMH7 & $1 \mathrm{U} 19$ & 95.8 & $\begin{array}{l}\text { Has insertion and highest } \\
\text { sequence similarity to suggested } \\
\text { template }\end{array}$ \\
\hline $\begin{array}{l}\text { Helix } \\
8\end{array}$ & $1 \mathrm{U} 19$ & 100.0 & $\begin{array}{l}\text { Highest sequence similarity to } \\
\text { suggested template }\end{array}$ \\
\hline
\end{tabular}

Further the sequence of amino acid for the apoprotein was also compared with the known structured sequence using PDB BLAST (Altschul et.al., 1990). The result signifies that the co-crystallized structure of Rhodopsin from organism Bos taurus (PDB ID-1U19) had the best sequence identity (83\%) and had no gaps. After many rounds of loop refinement, an optimum structure was obtained (Fig 1). The arrangement of the phi and psi angles for the amino acid residues was presented in Fig. 2 A (Table 2). The percentage of phi and psi angles in the favorably allowed region was $88.6 \%$ and none of the residues was located in the prohibited region. The considered mean square root deviation among the intended model and template structure was found to be $0.213 \AA$ (Fig. $2 \mathrm{D})$. The Z-scores of the model and the template were 3.5 and -4.11 validated the quality of the model. Comparative study of the model and the guided structure shows similar profiles, as seen in Fig. 2B, C.

Table 2 . Ramachandran plot statistics for the 3D model of rhodopsin, calculated using PROCHECK

\begin{tabular}{ll}
\hline Residues in most favoured regions & $88.6 \%$ \\
Residues in additional allowed regions & $11.1 \%$ \\
Residues in generously allowed regions & $0.3 \%$ \\
Residues in disallowed regions & $0.0 \%$ \\
\hline
\end{tabular}




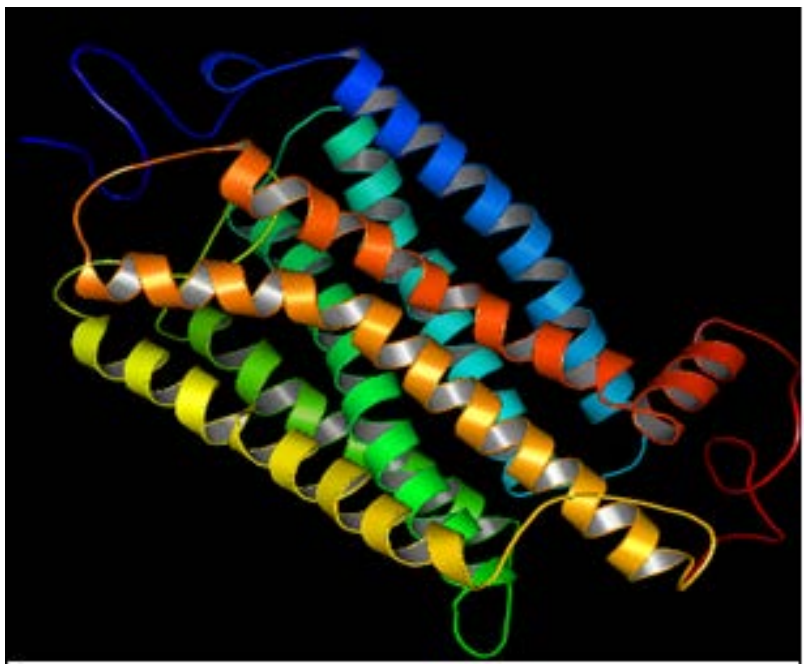

Figure 1.Using Bos taurus sequence as the template, the 3D model was built using MODELLER. Extensive loop refinements were carried out as it was found through the Ramachandran plot that residues in the loops were present in the disallowed region. Thus, after many rounds of loop refinement an optimum structure was obtained. In the image, blue represents the template and orange represents the structure of rhodopsin.
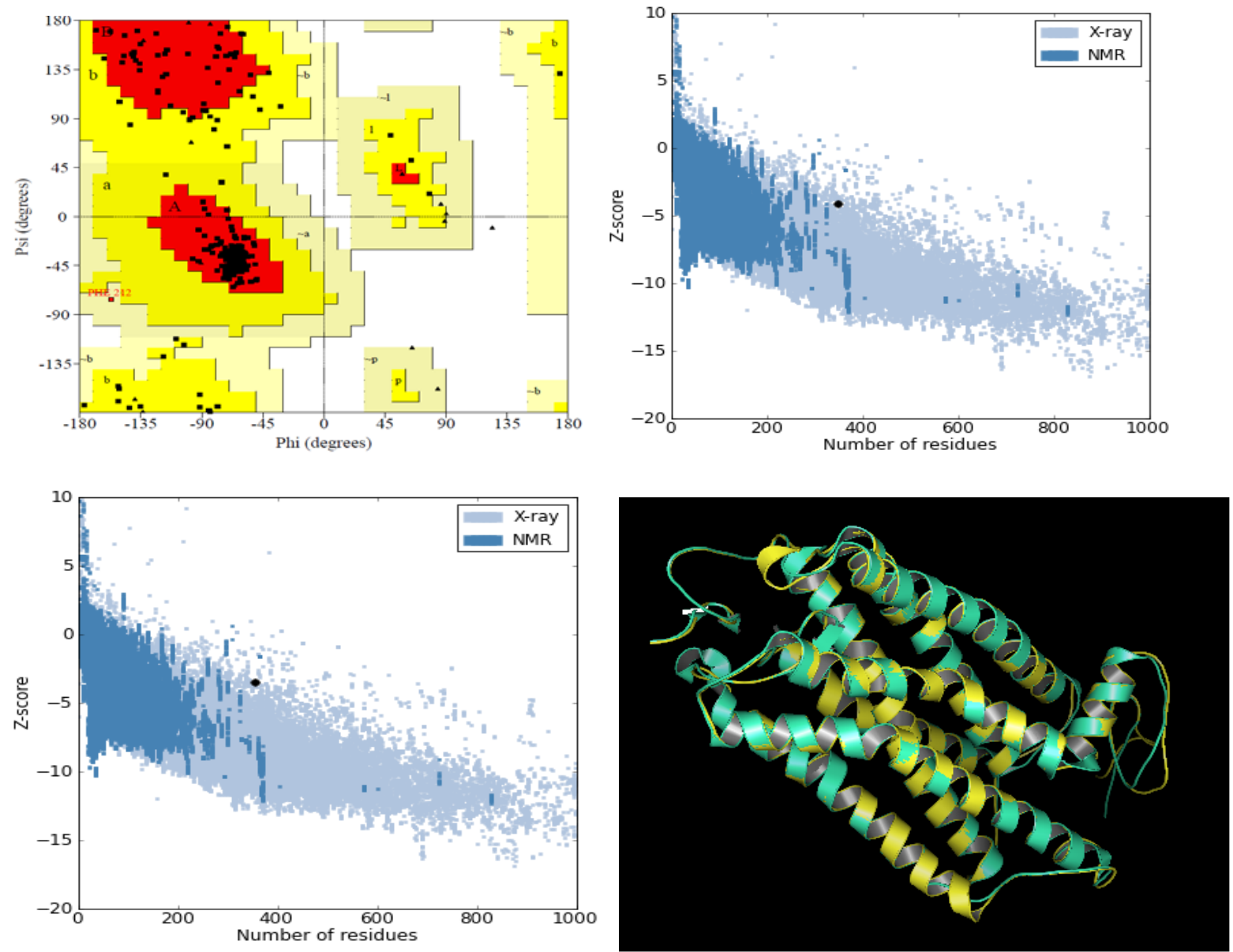

Plate 2. A. Ramachandran plot for the modeled structure of apoprotein from organism D. rerio. Red color represents most favored region. Additional allowed, generously allowed and disallowed regions are represented by yellow, light yellow and white colors respectively. B. and C: The plot contains the Z-scores of all experimentally determined protein chains in the current PDB that have been solved by either $\mathrm{X}$-ray diffraction or NMR. The plot is used to check whether the Z-score of the 3D structure is within the range of scores typically found for native proteins of a similar size. The Z-score of -4.11 and -3.5 (fig: $2 \mathrm{~B}$ and $2 \mathrm{C}$ respectively) represents the overall quality of the template structure and the target respectively.

\subsection{Covalent docking}

Covalent docking was performed using the prime Schrodinger. Apoprotein opsin is covalently attached to the chomatophore 11 cis retinal to obtain the structure of rhodhopsin and in porphyrosin, 11 cis 3,4 , di- dehydroretinol is the chromatophore that is attached to the apoprotein. In Rhodopsin and Porphyropsin, the chromophores are covalently attached to opsin through the nitrogen of Lysine 296 (Fig 3 A and Fig 3 B). On comparing the residues that are involved in the binding of 
11-cis-retinal and 11-cis 3, 4-didehydroretinal, residues like Glutamic acid 113, Alanine 117, Threonine 118, Glycine 121, Glutamic acid 122, Serine 186, Cysteine 187, Tyrosine 191, Methionine 207, Histidine 211, Phenylalanine 212, Phenylalanine 261, Trptophan 265, Tyrosine 268, Alanine 269, Alanine 292, Lysine 296 were found to be common (Fig. 3 C, D). Out of all these residues, lysine 296 is covalently attached and rests are encompassed in hydrophobic interactions with both the chromophores. The alignment of the template and the covalently docked structures (Rhodopsin and Porphyropsin) were checked and confirmed. It was found that the chromophores were bound in a similar fashion as that in the template (Fig $3 \mathrm{E}, \mathrm{F}$ ). The considered RMSD between Rhodopsin and the template was found to be $0.299 \AA$ and the root mean square deviation between Porphyropsin and the model template was $0.302 \AA$. The backbone atoms were considered for these calculations. These results indicate great similarity of the structure with that of the template and can be used for further analysis. It is also important to mention that zebra fish rhodopsin shares $\sim 80.4 \%$ homology to the human rhodopsin (accession P08100). Therefore, it may serve as an excellent model for the study of human diseases.
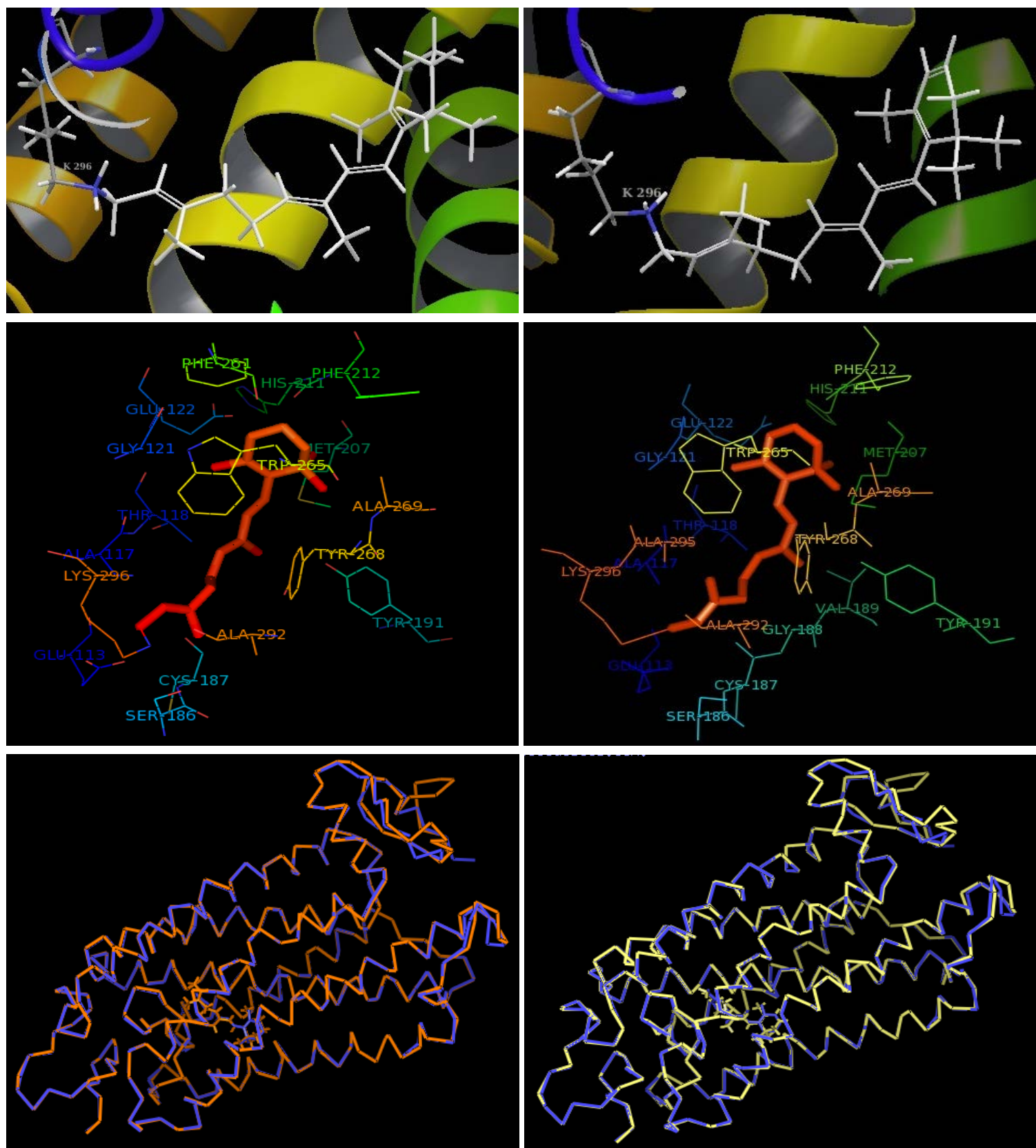

Figure 3. A, B Covalent binding of the chromophore 11-cis-retinal and 11-cis 3, 4-didehydroretinal to apoprotein Opsin through nitrogen of lysine 296 to obtain structures of rhodopsin and porphyropsin respectively. $\mathbf{C}$ and $\mathbf{D}$ show the amino acids that are involved in the binding of the chromophores, 11-cis-retinal and 11-cis 3, 4-didehydroretinal in rhodopsin and porphyropsin respectively. E The image of superimposed template and rhodopsin generated using PyMol. In the image, blue represents the template and orange represents the structure of rhodopsin. It can be observed that the 11-cis-retinal aligns in a similar fashion as in the template. F The image of superimposed template and porphyropsin generated using PyMol. In the image, blue represents the template and yellow represents the structure of porphyropsin. It can be observed that the 11-cis 3, 4-didehydroretinal aligns in a similar fashion as that of retinal in the template. 


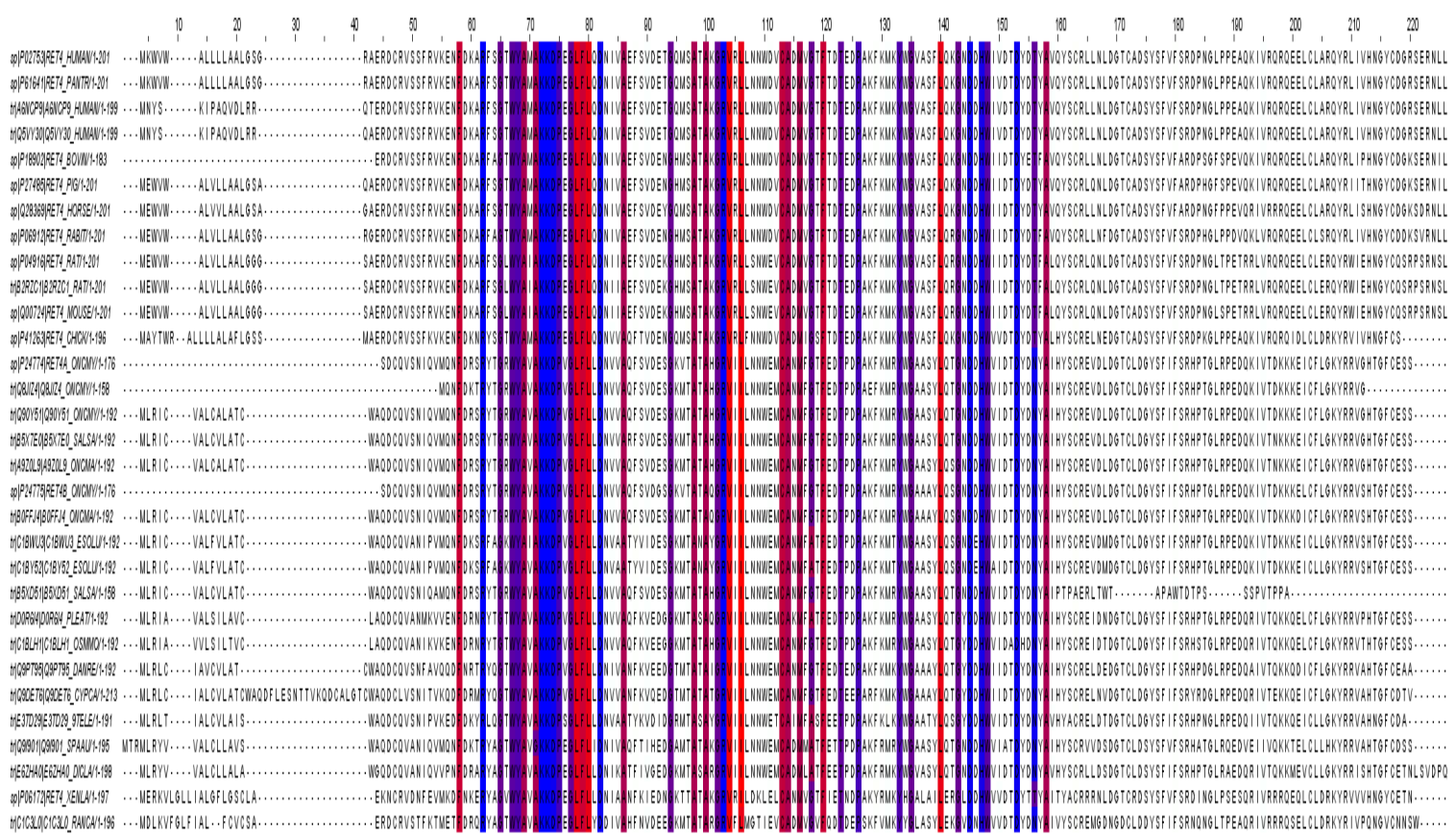

Figure 4. Sequence alignment of retinol binding proteins belonging to Lipocalin family. The residues showing $100 \%$ conservation are highlighted.

\section{Conclusion}

The physiochemical characteristics provided in depth perception about the nature of the apoprotein. Validation and evaluation of 3-D structure of the apoprotein show that the projected model is reliable and is reasonable at the present level of theory. Covalent docking of chromophores 11-cis-retinal and 11-cis 3, 4-didehydroretinal to lysine 296 resulted in the structures of Rhodopsin and Porphyropsin respectively. The predicted 3D structures and the residues involved in the binding of ligand can be used for guiding structural site-directed mutagenesis investigation. The structures may be used further to predict the key residues in the active site of the enzyme. Further, it can be used in understanding the structure - function relationships, and subsequently gain insight into its catalytic mechanism.

\section{Acknowledgement}

Sincere gratitude is due to Lt. Prof. U.C. Goswami for his kind guidance and for enabling us to accomplish our work methodically.

No financial support was received for the research, authorship, and/or publication of this current article.

\section{References}

Allison WT, Haimberger TJ, Hawryshyn CW, Temple SE. 2004. Visual pigment composition in zebrafish: evidence for a rhodopsin-porphyropsin interchange system. Vis Neurosci, 21: 945-952.

Altschul SF, Gish W, Miller W, Myers EW, Lipman DJ. 1990. Basic local alignment search tool. $J$ Mol Biol, 215: 403-410.

Ashokan KV, Mundaganur DS, Mundaganur YD. 2010. Catalase: Phylogenetic characterization to explore protein cluster. $J$ of Res in Bioinfo,1:1-8.
Cameron DA 2002. Mapping absorbance spectra, cone fractions, and neuronal mechanisms to photopic spectral sensitivity in the zebrafish. Vis Neurosci, 19: 365-372.

Chinen A, Hamaoka T, Yamada Y, Kawamura S 2003. Gene duplication and spectral diversification of cone visual pigments of zebrafish. Genetics, 163: 663-675.

Corush, J.B., 2019. Evolutionary patterns of diadromy in fishes: more than a transitional state between marine and freshwater. BMC Evolutionary Biology, 19(1):.168.

Enright, J.M., Toomey, M.B., Sato, S.Y., Temple, S.E., Allen, J.R., Fujiwara, R., Kramlinger, V.M., Nagy, L.D., Johnson, K.M., Xiao, Y. and How, M.J., 2015. Cyp27c1 red-shifts the spectral sensitivity of photoreceptors by converting vitamin A1 into A2. Curr Biol, 25(23): 3048-3057.

Gamage DG, Gunaratne A, Periyannan GR, Russell TG. 2019. Applicability of instability index for in vitro protein stability prediction. Protein Peptide Lett, 26:339-347.

Ganong WF 2005. Dynamics of blood and lymph flow. Review of Medical Physiology 22: 444-448.

Gasteiger E, Gattiker A, Hoogland C, Ivanyi I, Appel RD, Bairoch A 2003. ExPASy: the proteomics server for in-depth protein knowledge and analysis. Nucleic Acids Res, 31: 3784-3788.

Gestri, G., Link, B.A. and Neuhauss, S.C., 2012. The visual system of zebrafish and its use to model human ocular diseases. Dev Neurobiol, 72(3): 302-327.

Goldsmith P, Harris W 2003. The zebrafish as a tool for understanding the biology of visual disorders. Semin Cell Dev Biol, 14: 11-18.

Korenyak, D.A. and Govardovskii, V.I. 2013. Photoreceptors and visual pigments in three species of newts. $J$ Evol Biochem Physiol, 49(4): 399-407.

La Frano, M.R., Cai, Y., Burri, B.J. and Thilsted, S.H., 2018. Discovery and biological relevance of 3, 4-didehydroretinol (vitamin A2) in small indigenous fish species and its potential as a dietary source for addressing vitamin A deficiency. Int J Food Sci Nutr, 69(3): 253-261. 
Laskowski RA, MacArthur MW, Moss DS, Thornton JM. 1993. PROCHECK: a program to check the stereochemical quality of protein structures. J Appl Crystallogr, 26: 283-291.

Marschall, S., Pedersen, C. and Andersen, P.E., 2012. Investigation of the impact of water absorption on retinal OCT imaging in the $1060 \mathrm{~nm}$ range. Biomed Optics Exp, 3(7):16201631.

Morrow JM, Chang BS. 2015. Comparative mutagenesis studies of retinal release in light-activated zebrafish rhodopsin using fluorescence spectroscopy. Biochemistry, 54(29):4507-18.

Ochuko, J.E., Ovie, K.S. and Faith, I.I. 2014. Serum enzymes as a result of Azadirachta indica extract injection to African catfish Clarias gariepinus (Burchell, 1822). Jordan J Biol Sci, 147(1573): $1-5$

Porterfield JZ, Zlotnick A. 2010. A simple and general method for determining the protein and nucleic acid content of viruses by UV absorbance. Virology, 407(2):281-288.

Reddy, P. and Vanga, U.M.R., 2017. Homology Modeling and In silico Docking Studies of DszB Enzyme Protein, Hydroxyphenyl Benzene Sulfinate Desulfinase of Streptomyces sp. VUR PPR 101 Jordan J Biol Sci, 10(4): 309-316

Sahay A, Shakya M. 2010. In silico analysis and homology modelling of antioxidant proteins of spinach. J Proteomics Bioinform, 3:148-154.
Šali A, Blundell TL. 1993. Comparative protein modelling by satisfaction of spatial restraints. J Mol Biol, 234: 779-815.

Sharma A, Singla D, Rashid M, Raghava GP. 2014. Designing of peptides with desired half-life in intestine-like environment. BMC Bioinformatics, 15:282-287.

Toyama, M., Hironaka, M., Yamahama, Y., Horiguchi, H., Tsukada, O., Uto, N., Ueno, Y., Tokunaga, F., Seno, K. and Hariyama, T., 2008. Presence of rhodopsin and porphyropsin in the eyes of 164 fishes, representing marine, diadromous, coastal, and freshwater species-a qualitative and comparative study. Photochem, 84(4): 996-1002.

Wanyonyi SS, Sharp JA, Khalil E, Lefevre C, Nicholas KR. 2011. Tammar wallaby mammary cathelicidins are differentially expressed during lactation and exhibit antimicrobial and cell proliferative activity. Comp Biochem Physiol Part A Mol Integr Physiol. 160(3):431-439.

Wiederstein M, Sippl MJ. 2007. ProSA-web: interactive web service for the recognition of errors in three-dimensional structures of proteins. Nucleic Acids Res, 35: 407-410.

Worth CL, Kreuchwig A, Kleinau G, Krause G. 2011. GPCRSSFE: a comprehensive database of G-protein-coupled receptor template predictions and homology models. BMC Bioinformatics, 12:185-198. 